\title{
Dorsal Dislocation of Medial Cuneiform Along with Lisfranc Injury: Approach to a Rare Injury
}

\section{Kumar MA* and Madaan E}

Guru Teg Bahadur Hospital, Delhi, India

*Corresponding author: Mohith Kumar Arora, Guru Teg Bahadur Hospital, Delhi, India, Tel: 9013490058; E-mail: drmkarora@yahoo.com

Received date: Feb 03, 2017; Accepted date: Feb 16, 2017; Published date: Feb 20, 2017

Citation: Kumar MA, Madaan E (2017) Dorsal Dislocation of Medial Cuneiform along with Lisfranc Injury: approach to a Rare Injury. J Clin Exp Orthop 3: 30. doi:10.4172/2471-8416.100030

Copyright: (c) 2017 Kumar MA, et al. This is an open-access article distributed under the terms of the Creative Commons Attribution License, which permits unrestricted use, distribution, and reproduction in any medium, provided the original author and source are credited.

\section{Abstract}

Isolated medial cuneiform dislocation along with lisfranc dislocation is a very rare injury. A case report of this type of injury is presented to highlight the rarity of the injury and to emphasize the importance of thorough clinico-radiological examination to aid in the diagnosis. Open reduction and internal fixation is the treatment of choice.

\section{Keywords: Dislocation; Medial cuneiform; Lisfranc}

\section{Introduction}

The medial cuneiform articulates with navicular and first metatarsal. It is the largest of all the three cuneiform bones. Together with the first and second metatarsals, the navicular, and the intermediate cuneiform, this bone is part of joints in the mid-foot. Medial cuneiform serves as site of attachment for numerous ligaments, peroneus longus and tibialis anterior muscles. Medial cuneiform also forms a part of medial longitudinal arch of the foot. Dorsal dislocation of the medial cuneiform is very rare injury. We present a case report of dorsal dislocation of medial cuneiform along with lisfranc injury in a 20 year old male.

\section{Case Report}

A 20 year old male presented to the department of orthopaedics in GTB hospital with pain and swelling Right foot. His right foot got stuck under the brakes of the four wheeler he was driving. As a result of which there was sudden plantar flexion of the affected foot. On examination there was swelling and tenderness of Right foot. There were abrasions present on the dorsum of the foot. There was no distal neuro-vascular deficit. $X$ rays (Figure 1 ) showed dorsal dislocation of medial cuneiform along with dorsal displacement of second metatarsal (Figure 1).

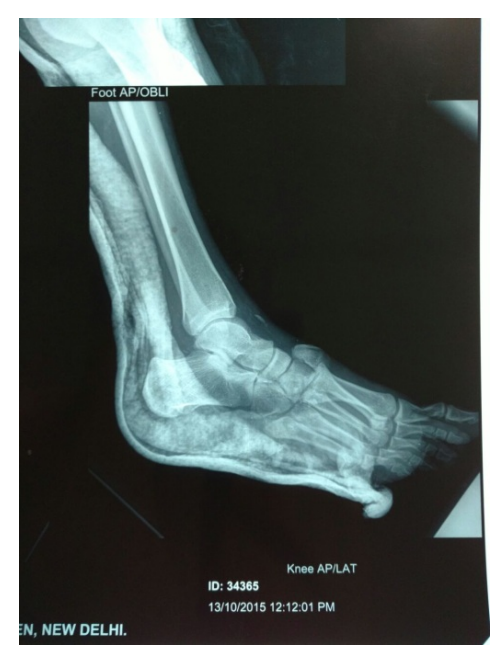

Figure 1: X ray showing dorsal dislocation of medial cuneiform.

CT scan (Figure 2) was done to rule out injuries to other bones. The patient was advised strict limb elevation and ice fomentation to prevent compartment syndrome. The patient was operated $24 \mathrm{~h}$ after the injury. The open reduction was done by giving incision between first and second metatarsal (Figure 2).

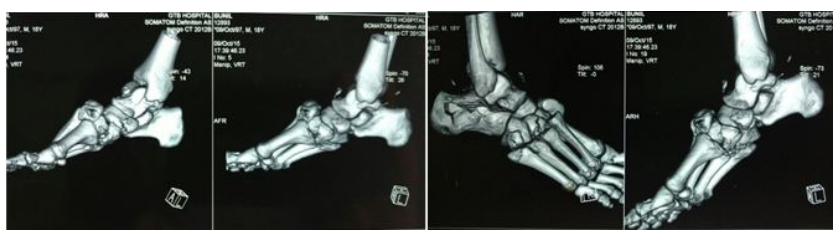

Figure 2: CT scan film with $3 \mathrm{D}$ reconstruction showing medial cuneiform dislocation along with Lisfranc injury.

Per operatively dorsal dislocation of medial cuneiform (Figure 3) was present with gap present between navicular and first metatarsal. The reduction of medial cuneiform and lisfranc joint was done and fixed with k-wires. Post-operative period was uneventful. K-wires were removed at 6 weeks following which 
weight bearing was started. At 1 year follow up patient is asymptomatic (Figures 3 and 4).

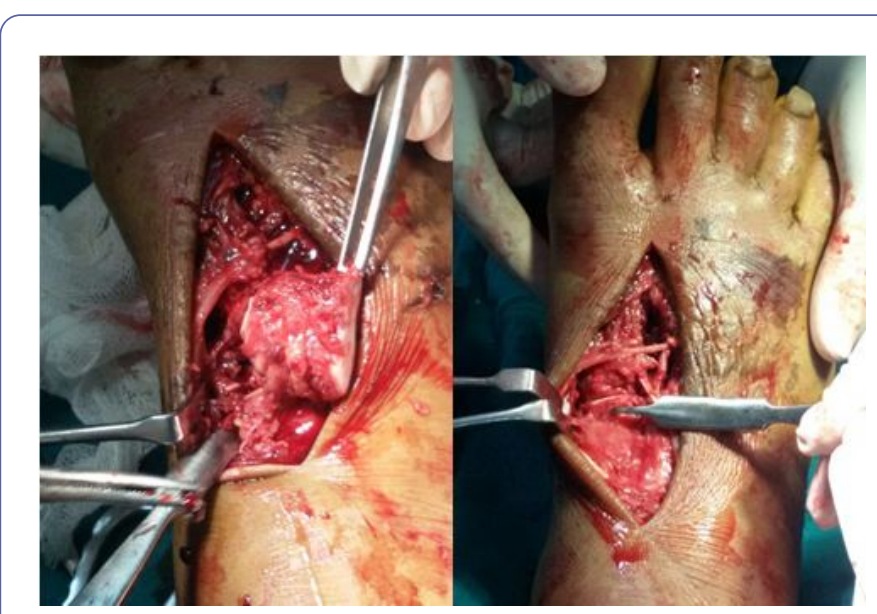

Figure 3: Per op pics showing the dislocated medial cuneiform and after reduction of medial cuneiform.

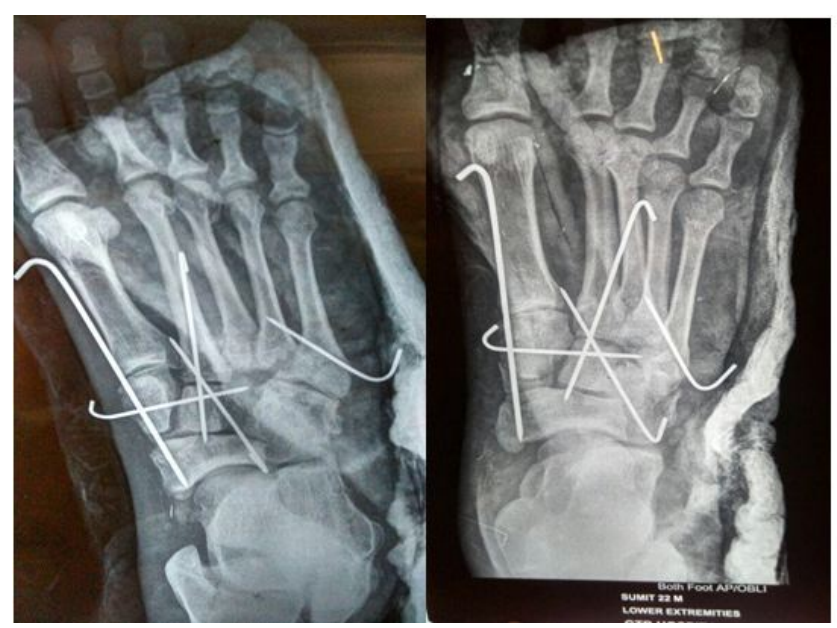

Figure 4: Post op $\mathrm{x}$ rays showing multiple $\mathrm{k}$ wires used for reduction.

\section{Discussion}

All the three cuneiforms are wedge shaped and forms part of the transverse and medial longitudinal arch of the foot. The medial, intermediate and lateral cuneiform articulates distally with first, second and third metatarsals respectively. While they articulate with navicular bone proximally [1]. The intercuneiform joints are planar-type joints that permit only gliding and rotation during pronation and supination movements [2]. The stability of these bones is achieved by the deep transverse, dorsal, and plantar ligaments. However in cases of extreme plantar flexion these ligament tear apart leading to dorsal dislocation of cuneiforms. The literature search shows few case reports of dislocation of medial cuneiform [3-6]. The presence of lisfranc injury along with dorsal dislocation of medial cuneiform makes this a rare combination which has not been reported yet to our knowledge.

In our case the sequence of events leading to dislocation of medial cuneiform can be explained as follows. The patient foot got plantar flexed forcefully when it got stuck under the brakes of the vehicle he was driving. Attempting dorsiflexion of the plantar flexed foot led to disruptions of ligaments around medial cuneiform leading to its dorsal dislocation. Additionally the pull of tibialis anterior during attempt of dorsiflexion might have also contributed to medial cuneiform dislocation. This mechanism also explains dorsal dislocations of other metatarsals leading to concomitant lisfranc injury. Careful watch should be done to prevent the development of compartment syndrome of foot. Closed reduction can be attempted to reduce the dislocation but is usually unsuccessful because of the swelling, hence open reduction is the treatment of choice in these types of cases [6].

\section{Conclusion}

Dorsal dislocation of medial cuneiform along with lisfranc injury is a rare combination. Thorough clinico-radiological examination should be done to detect these types of injuries. Open reduction is the gold standard treatment for these injuries.

\section{References}

1. JS Early (2001) "Cuneiform injuries," in Rockwood and Green's Fractures in Adults. Rockwood CA, Green DP, Heckman JD, and Bucholz RW, Eds, Lippincott Williams \& Wilkins, Philadelphia, USA. pp. 2199-2202.

2. H. Gray (1989) Anatomy of the Human Body, Churchill Livingstone, Edinburgh, Scotland.

3. Aitken SA, Shortt N (2012) Dorsomedial fracture dislocation of the first and medial cuneiform: a case report. J Foot Ankle Surg 51: 795-797.

4. Bulut G, Yasmin D, Hebeli N (2009) A complex variant of Lisfranc joint complex injury. J Am Podiatr Med Assoc 99: 359-363.

5. Levine BP, Stoppacher R, Kristiansen TK (1998) Plantar lateral dislocation of the medial cuneiform: a case report. Foot Ankle Int 19: 118-119.

6. Hidalgo-Ovejero AM, Garcia-Mata S (2005) Complete medial dislocation of the first cuneiform: a case report. J Foot Ankle Surg 44: 478-482. 